\title{
Equilibration dynamics of wetting layers
}

\author{
H. Musil, S. Herminghaus * and P. Leiderer \\ Fakultät für Physik, Universilät Konstanz, 7750 Konstanz, Germany
}

Received 17 May 1993; accepted for publication 21 June 1993

A direct and quantitative investigation of the transport kinetics of wetting layers is presented. The temporal decay of pulsed laser induced spatial thickness modulations in liquid ethanol films on a silver surface is monitored by means of optically excited surface plasmons. Our method allows to distinguish between different equilibration mechanisms. Under the conditions in our experiments. the dominant transport process is direct exchange of molecules with the saturated vapor. Contributions from lateral diffusion were found to be less than $10^{-8} \mathrm{~cm}^{2} / \mathrm{s}$.

Thin films on solid surfaces have become one of the most attractive fields of condensed matter physics. This is not only due to their technological importance. It is also for the sake of the rich and fascinating dynamical phenomena discovered in surface diffusion [1], catalytic surface reactions [2] and the dynamics of wetting far from thermodynamical equilibrium [3-5]. For specific investigations of these phenomena, one has to identify the basic transport mechanisms and determine the corresponding transport coefficients.

In submonolayer chemisorbed films, transport proceeds mainly by lateral diffusion of the adatoms or admolecules on the substrate. The diffusion coefficient $D$ is usually measured as follows [6]. By illuminating the sample with two interfering pulsed laser beams, a spatiahy periodic coverage modulation is induced due to laser induced thermal desorption. According to Fick's law, lateral diffusion leads to an exponential decay of the modulation amplitude, $a \propto \mathrm{e}^{-A t}$. The decay constant is given by $A=D q^{2}$, where $D$ is the diffusion coefficient to be measured and $q$ is the wave number of the coverage modulation which is on the order of $10^{6} \mathrm{~m}^{-1}$ in these experi-

\footnotetext{
* To whom correspondence should be addressed.
}

ments. The decay can be monitored by diffraction of a probe laser beam from the coverage modulation [7]. Although the diffracted intensity is rather low, it is sufficient for observing the slow (several minutes or hours) decay of modulations in chemisorbed systems.

If one considers physisorbed films such as wetting layers, whose thickness ranges from a few monolayers up to several $100 \AA$. one is faced with a much more complicated transport behavior, since capillary waves or direct exchange of molecules with the (saturated) vapor may play an important role. A spatially periodic thickness modulation is then expected to decay with a decay constant $A \propto q^{\prime \prime}$, where the value of $\nu$ is indicative of the dominant transport mechanism. For instance, direct exchange of molecules with the vapor phase leads to $\nu=0$, lateral diffusion within the film yields $\nu=2$, surface diffusion driven by the surface tension of the adsorbate yields $\nu=4$ [8]. Variation of $q$ and observation of the scaling behavior of $A(q)$ allows one to identify the dominant mechanism. Due to the low binding energy and the presence of several competing transport mechanisms in physisorbed films, the decay is expected to proceed much faster than in chemisorbed systems, being not readily observable with the above-mentioned diffraction technique. Consequently, a sensitive experimental 
method with superior temporal resolution is required.

It has already been shown that changes in the thickness of physisorbed films, as they occur in pulsed laser desorption experiments, are advantageously investigated by means of optically excited surface plasmons [9]. In these experiments, the substrate consists of a metal film (silver, in our case) evaporated onto the base of a glass prism. A light beam illuminates the metal from inside the prism under total internal reflection conditions, giving rise to an evanescent light wave which penetrates the metal film. At a particular angle of incidence, the phase velocity of the evanescent matches the velocity of the surface plasmons propagating on the free metal surface, which results in a pronounced attenuation of the reflected light due to resonant excitation of surface plasmons. The position of this resonance changes significantly if a transparent film is being adsorbed on the metal. If one measures the reflected intensity at an angle of incidence corresponding to one of the wings of the resonance, changes in the thickness of the adsorbed film will result in changes of the reflected light intensity. In this way, changes in film thickness on the order of a monolayer can easily be observed on a nanosecond time scale $[10,11]$.

The goal of the present work is to identify the transport mechanisms in wetting layers by observing the decay of pulsed laser induced thickness modulations. In order to provide the surface plasmons with the required spatial resolution, their intensity is spatially modulated with a period equal to that of the thickness modulation to be observed. Depending on the relative phase of the plasmon intensity pattern and the thickness modulation, the film thickness is measured in the "valleys" or on the "hills" of the thickness modulation. The difference of both thickness values yields the modulation amplitude.

The optical apparatus is depicted in fig. 1. An optical diffraction grating was imaged onto the sample, with a Q-switched Nd:YAG laser ( $\lambda=$ $532 \mathrm{~nm}, 7 \mathrm{~ns}$ pulse duration) and a $\mathrm{He}-\mathrm{Ne}$ laser $(\lambda=633 \mathrm{~nm}$, continuous wave) as light sources. The Nd: YAG laser served for the creation of the thickness modulation, the $\mathrm{He}-\mathrm{Ne}$ laser for its

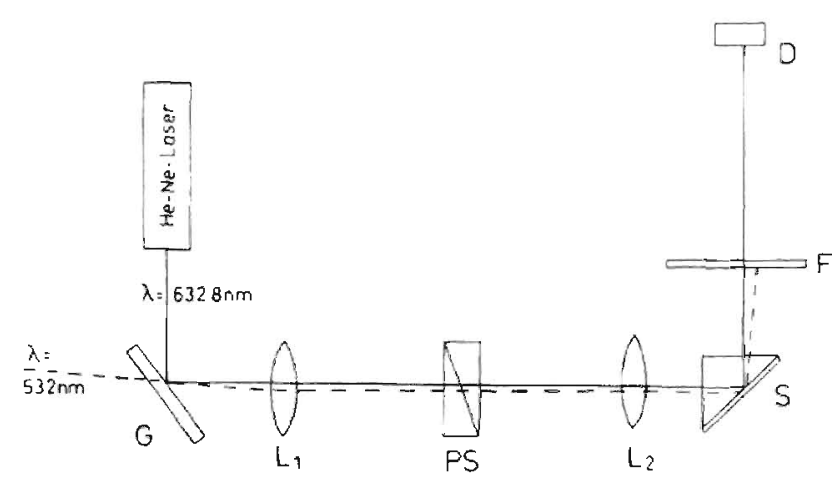

Fig. 1. Experimental setup. $G$, optical grating; $L_{1}, L_{2}$, lenses imaging the grating onto the sample; PS. phase shifter assembly: S. sample (the sample is mounted in an optical cryostat which is not shown); $F$, filter: $D$, detector. The solid line indicates the beam path of the probe ( $\mathrm{He}-\mathrm{Ne}$ ) laser, the dashed line represents the pulsed laser beam.

detection. In this way, perfect matching of the pulsed laser induced spatial thickness modulation and the intensity modulation of the probing surface plasmon field was achieved. For each wavelength, only two of the diffracted beams (zeroorder and one of the first-order beams) were allowed to reach the sample. In this way, an infinite focal depth image of the grating onto the sample was obtained.

The Nd:YAG laser beam was adjusted so as to meet the surface plasmon resonance angle in order to achieve efficient coupling of energy into the substrate. Since only the silver film is being heated significantly by the laser pulse, a strong temperature gradient into the glass prism builds up which provides fast recooling after the pulse. From the thermal properties of the materials involved, one can estimate that after approximately $40 \mu$ s the amplitude of the spatial temperature profile has decayed to less than $10 \mathrm{mK}$. The overall heating of the surface relaxes to less than $1 \mathrm{~K}$ above the equilibrium temperature within about $1 \mathrm{~ms}$.

The He-Ne laser beam was adjusted to the lower-angle wing of the surface plasmon resonance, yielding an approximately linear relationship between the measured intensity and the thickness of the wetting layer at places with appreciabe surface plasmon intensity. Consequently, a continuous shift of the surface plasmon 
intensity pattern with respect to the thickness modulation yields an intensity oscillation whose amplitude directly corresponds to the amplitude of the spatial film thickness modulation. This continuous shift is easily achieved by ramping the optical pathlength of one of the orders of diffraction of the probe laser beam, using a simple phase shifter assembly: two glass plates are turned about their vertical axis, in opposite directions to avoid parallel displacement of the beam.

Experiments were performed with wetting layers of ethanol adjacent to a vertical substrate. The sample consisted of a closed volume next to the silver-coated glass prism. When the cell was filled partly with ethanol (supplied by MERCK, p.a. grade), a wetting layer formed on the silver surface due to long-range interaction forces between the ethanol molecules and the walls. The film thickness could be measured by means of the surface plasmon resonance position to be $36 \pm 4$ $\AA$ under the conditions of our experiment.

Fig. 2 shows a typical intensity transient after operating the pulsed laser. The phase of the surface plasmon pattern excited by the $\mathrm{He}-\mathrm{Ne}$ laser is being changed continuously, giving rise to the oscillations whose envelope is a measure for

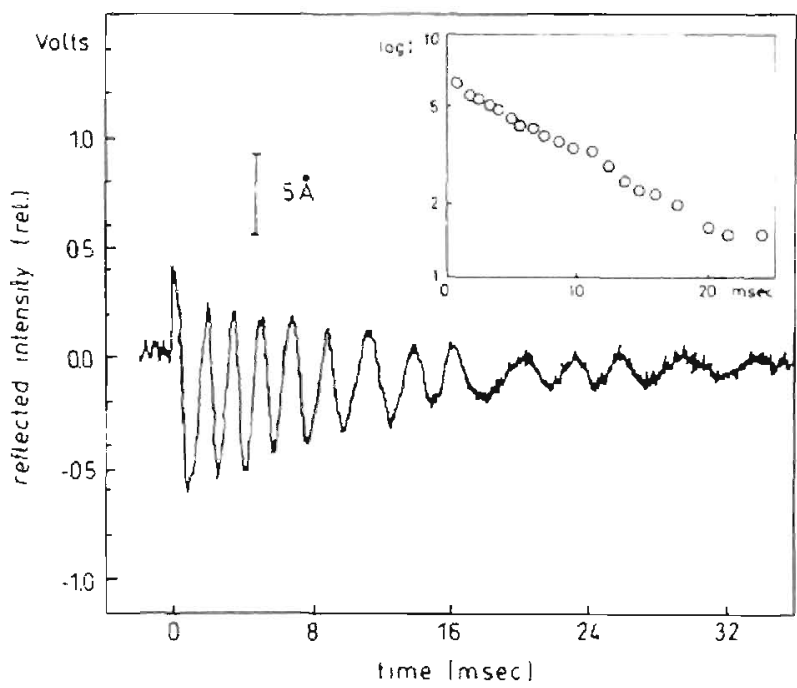

Fig. 2. Typical intensity transient after operating the pulsed laser, taken with ethanol wetting layer at a temperature of 190 $K$. The envelope of the oscillation yields the decay of the thickness modulation, which in this case proceeds within a few milliseconds. The inset shows a semi-log plot of the oscillation amplitude, demonstrating the exponential decay.

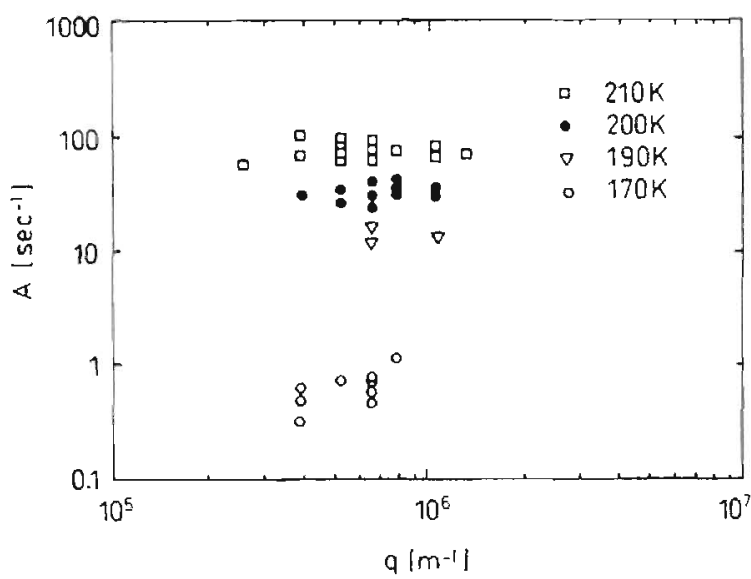

Fig. 3. The decay rate $A$ versus the wave number of the modulation, $q$. For fixed temperature, no significant dependence of $A$ on $q$ is observed.

the pulsed laser induced thickness modulation amplitude. Note that a temporal resolution of about one millisecond (the time elapsed between two intensity extrema) is achieved. The calibration of the amplitude can be obtained from the known surface plasmon resonance shift with adsorbed film thickness [12]. According to this calibration, the bar in fig. 2 would correspond to a corrugation amplitude of $2.5 \AA$. However, a rigorous treatment of surface plasmons propagating on a corrugated surface shows that actually the sensitivity is smaller by a factor of two, resulting in the calibration given in the figure [8]. A semilogarithmic plot of the envelope of the oscillation is given in the inset, showing a fairly exponential behavior.

The period of the spatial thickness modulation induced by the pulsed laser can easily be varied by exchanging the optical grating. Fig. 3 shows the temporal decay constant $A$ of the modulation as a function of its wave number for four different temperatures (above the melting point, $T_{\mathrm{m}}=$ $156 \mathrm{~K}$ [13]). No significant dependence on the wave number is observed (for $170 \mathrm{~K}$, only a slight tendency of an increasing decay constant may be present). Taking into account the $q$-dependences for the various decay processes mentioned above, this suggests that the liquid ethanol film equilibrates to the van der Waals equilibrium thickness by exchange of material with the saturated vapor rather than by lateral transport processes. From 


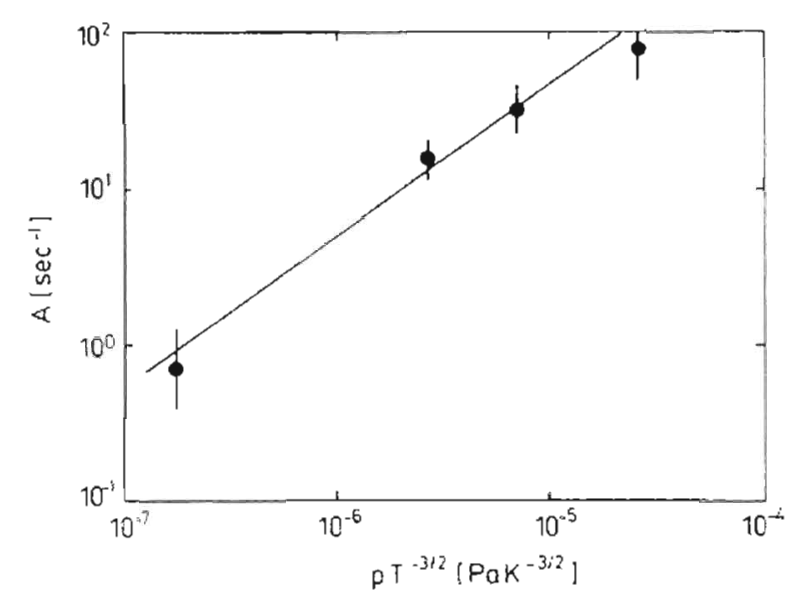

Fig. 4. The typical decay rates obtained for different temperatures. The straight line represents the equilibration rate expected from direct exchange with the vapor phase. The error bars correspond to the scattering of the data in fig. 3 .

the scattering of the data, we obtain an upper bound for contributions from lateral diffusion effects, amounting to $10^{-8} \mathrm{~cm}^{2} / \mathrm{s}$.

The dominance of exchange with the vapor phase can be corroborated as follows. From kinetic gas theory, we obtain for the adsorption rate of molecules from the gas phase onto the film surface

adsorption rate $=\frac{\beta}{\rho} \sqrt{\frac{3}{m}} \frac{\alpha}{d^{\gamma+1}} p T^{-3 / 2}$,

where $\beta$ is the sticking coefficient of the ethanol, $\rho$ is the liquid density, $m$ is the molecular mass, and $d$ is the film thickness. The parameters $\alpha$ and $\gamma$ characterize the liquid-substrate interaction potential. Accordingly, we plotted the average values af $A$, obtained at fixed temperatures, versus the quantity $p T^{-3 / 2}$ in fig. 4 . Satisfactory agreement is found with the straight line representing eq. (1). Although the appropriate value of $\gamma$ is presently under discussion [14,15], we shall, for a rough estimate, adopt its classical (van der Waals) value $\gamma=3$ [16]. Under this assumption, we obtain from our data $\alpha \beta \approx 1.9 \times 10^{5} \mathrm{~K} \AA^{3}$. The sticking coefficient is known to be $\beta \approx 0.04$ [17], so we obtain the van der Waals constant $\alpha \approx 4.8 \times 10^{6} \mathrm{~K}^{3}$.
Van der Waals constants are known only for few wetting film systems. For comparison with our value, we take the van der Waals constant for neon films on the same substrate (silver/glass), which is $1.37 \times 10^{5} \mathrm{~K} \AA^{3}$ [18]. The binding energy due to long-range forces seems to be much stronger for ethanol. This is not surprising since the molecolar polarizability of ethanol is about one order of magnitude larger than that of neon [19]. Furthermore, the finite dipole moment of the ethanol molecule gives rise to additional attractive long-range interactions with the substrate. It will be of particular interest to investigate the lower-temperature regions where equilibration by exchange with the vapor is less pronounced (due to the low gas density). Effects like surface melting or surface roughening should show up in the surface diffusion contribution to the decay.

In conclusion, we have introcuced a novel optical method of observing the transport kinetics in wetting layers. It allows to monitor small-scale thickness variations with millisecond temporal resolution. By varying the length scale of observation, we can distinguish between different transport mechanisms. We showed that for wetting layers of ethanol on a silver surface, equilibration proceeds via direct exchange of material with the vapor phase.

We appreciate inspiring discussions with Uwe Albrecht and Thomas Paatzsch. This work was supported by the Deutsche Forschungsgemeinschaft (SFB 306).

\section{References}

[1] X.D. Zhu, A. Lee, A. Wong and U. Linke, Phys. Rev. Lett. 68 (1992) 1862.

[2] S. Jakubith, H.H. Rotermund, W. Engel, A. von Oertzen and G. Ertl, Phys. Rev. Lett. 65 (1990) 3013.

[3] P. Guenoun, D. Beysens and M. Robert, Phys. Rev. Lett. 65 (1989) 2406.

[4] G. Reiter, Phys. Rev. Lett. 68 (1992) 75.

[5] J.B. Brzoska, F. Brochard-Wyart and F. Rondelez, Europhys. Lett. 19 (1992) 97.

[6] X.D. Xiao, Y. Xie and Y.R. Shen, Surf. Sci. 271 (1992) 295.

[7] X.D. Zhu, Th. Rasing and Y.R. Shen, Phys. Rev. Lett. 61 (1988) 2883. 
[8] Theoretical details will be presented in a forthcoming publication.

[9] S. Puderbach, S. Herminghaus and P. Leiderer, Phys. Lett. A 130 (1988) 401.

[10] S. Herminghaus and P. Leiderer, Appl. Phys. Lett. 58 (1991) 352

[11] S. Herminghaus and P. Leiderer, Proc. SPIE 1594 (1991) 334.

[12] 1. Pockrand, Surf. Sci. 72 (1978) 577.

[13] K. Schäfer and E. Lax, Eds., Landoldt-Börnstein; Zahlenwerte und Funktionen, Part II, Vol. 2a (Berlin, 1961).
[14] D. Beaglehole, E.Z. Radlinska, B.W. Ninham and H.K. Christenson, Phys. Rev. Lett. 66 (1991) 2084.

[15] P.S. Vukusic, G.W. Bradberry and J.R. Sambles, Surf. Sci. Lett. 277 (1992) L34.

[16] 1.E. Dzyaloshinskii, E.M. Lifshitz and L.P. Pitaevskii, Adv. Phys. 10 (1961) 165.

[17] Van P. Carey, Liquid-Vapor Phase Change Phenomena (Hemisphere Publishing, Washington, 1992).

[18] U. Albrecht, PhD Thesis, Konstanz, Germany, 1992.

[19] R.C. Weast, Ed. CRC Handbook of Chemistry and Physics (CRC Press, Boca Raton, FL, 1987). 